\title{
Refinements of the integral form of Jensen's and the Lah-Ribarič inequalities and applications for Csiszár divergence
}

\section{J. Pečarić ${ }^{1}$ and J. Perićć ${ }^{*}$}

"Correspondence: jperic@pmfst.hr 2Department of Mathematics, Faculty of Science, University of Split, Split, Croatia

Full list of author information is available at the end of the article

\begin{abstract}
In this paper, we give refinements of the integral form of Jensen's inequality and the Lah-Ribarič inequality. Using these results, we obtain a refinement of the Hölder inequality and a refinement of some inequalities for integral power means and quasiarithmetic means. We also give applications in information theory, namely, we give some interesting estimates for the integral Csiszár divergence and its important particular cases.
\end{abstract}

MSC: 26D15;94A15

Keywords: Jensen's inequality; Lah-Ribarič inequality; Hermite-Hadamard inequality; Hölder inequality; Csiszár divergence; Integral form

\section{Introduction}

Let $I$ be an interval in $\mathbb{R}$, and let $f: I \rightarrow \mathbb{R}$ a convex function. If $\boldsymbol{x}=\left(x_{1}, \ldots, x_{n}\right)$ is any $n$ tuple in $I^{n}$ and $\boldsymbol{p}=\left(p_{1}, \ldots, p_{n}\right)$ a nonnegative $n$-tuple such that $P_{n}=\sum_{i=1}^{n} p_{i}>0$, then the well-known Jensen inequality

$$
f\left(\frac{1}{P_{n}} \sum_{i=1}^{n} p_{i} x_{i}\right) \leq \frac{1}{P_{n}} \sum_{i=1}^{n} p_{i} f\left(x_{i}\right)
$$

holds (see [6] or, e.g., [16, p. 43]). If $f$ is strictly convex, then (1.1) is strict unless $x_{i}=c$ for all $i \in\left\{j: p_{j}>0\right\}$.

Jensen's inequality is probably the most important inequality: it has many applications in mathematics and statistics, and some other well-known inequalities are its particular cases (such as Cauchy's inequality, Hölder's inequality, A-G-H inequality, etc.).

One of many generalizations of the Jensen inequality is its integral form (see [1,7], or, e.g., $[8])$.

Theorem 1.1 (Integral form of Jensen's inequality) Let $:[a, b] \rightarrow \mathbb{R}$ be an integrable function, and let $p:[a, b] \rightarrow \mathbb{R}$ be a nonnegative function. If $f$ is a convex function given on an

(c) The Author(s) 2020. This article is licensed under a Creative Commons Attribution 4.0 International License, which permits use, sharing, adaptation, distribution and reproduction in any medium or format, as long as you give appropriate credit to the original author(s) and the source, provide a link to the Creative Commons licence, and indicate if changes were made. The images or other third party material in this article are included in the article's Creative Commons licence, unless indicated otherwise in a credit line to the material. If material is not included in the article's Creative Commons licence and your intended use is not permitted by statutory regulation or exceeds the permitted use, you will need to obtain permission directly from the copyright holder. To view a copy of this licence, visit http://creativecommons.org/licenses/by/4.0/. 
interval I that includes the image of $g$, then

$$
f\left(\frac{1}{P(b)} \int_{a}^{b} p(t) g(t) d t\right) \leq \frac{1}{P(b)} \int_{a}^{b} p(t) f(g(t)) d t
$$

where

$$
P(t)=\int_{a}^{t} p(x) d x
$$

Our first main result is a refinement of inequality (1.2).

Strongly related to Jensen's inequality is the Lah-Ribarič inequality (see [11] or, e.g., [13, p. 9]). Its integral form is given in the following theorem.

Theorem 1.2 (Integral form of the Lah-Ribarič inequality) Let $g:[a, b] \rightarrow \mathbb{R}$ be an integrable function such that $m \leq g(t) \leq M$ for $t \in[a, b], m<M$, and let $p:[a, b] \rightarrow \mathbb{R}$ be a nonnegative function. If $f$ is a convex function given on an interval I such that $[m, M] \subseteq I$, then

$$
\frac{1}{P(b)} \int_{a}^{b} p(t) f(g(t)) d t \leq \frac{M-\bar{g}}{M-m} f(m)+\frac{\bar{g}-m}{M-m} f(M),
$$

where $P$ is given as before, and

$$
\bar{g}=\frac{\int_{a}^{b} p(t) g(t) d t}{P(b)}
$$

Our second main result is a refinement of inequality (1.3).

Another famous inequality established for the class of convex functions is the HermiteHadamard inequality. This double inequality, which was first discovered by Hermite in 1881 , is stated as follows (see, e.g., [16, p. 137]). Let $f$ be a convex function on $[a, b] \subset \mathbb{R}$, where $a<b$. Then

$$
f\left(\frac{a+b}{2}\right) \leq \frac{1}{b-a} \int_{a}^{b} f(x) d x \leq \frac{f(a)+f(b)}{2} .
$$

This result was later incorrectly attributed to Hadamard, who apparently was not aware of Hermite's discovery, and today, when relating to (1.4), we use both names.

This result can be improved by applying (1.4) on each subinterval $\left[a, \frac{a+b}{2}\right],\left[\frac{a+b}{2}, b\right]$, and the following result is obtained (see [14, p. 37]):

$$
f\left(\frac{a+b}{2}\right) \leq l \leq \frac{1}{b-a} \int_{a}^{b} f(x) d x \leq L \leq \frac{f(a)+f(b)}{2}
$$

where $l=\frac{1}{2}\left(f\left(\frac{3 b+a}{4}\right)+f\left(\frac{b+3 a}{2}\right)\right)$ and $L=\frac{1}{2}\left(f\left(\frac{b+a}{2}\right)+\frac{f(a)+f(b)}{2}\right)$.

The following improvement of (1.5) is given in [2].

Theorem 1.3 Let $f: I \rightarrow \mathbb{R}$ be a convex function on $I$. Then for all $\lambda \in[0,1]$ and $a, b \in I$, we have

$$
f\left(\frac{a+b}{2}\right) \leq l(\lambda) \leq \frac{1}{b-a} \int_{a}^{b} f(x) d x \leq L(\lambda) \leq \frac{f(a)+f(b)}{2}
$$


where

$$
l(\lambda)=\lambda f\left(\frac{\lambda b+(2-\lambda) a}{2}\right)+(1-\lambda) f\left(\frac{(1+\lambda) b+(1-\lambda) a}{2}\right)
$$

and

$$
L(\lambda)=\frac{1}{2}(f(\lambda b+(1-\lambda) a)+\lambda f(a)+(1-\lambda) f(b)) .
$$

Inequality (1.6) for $\lambda=\frac{1}{2}$ gives inequality (1.5). Further improvement was given in [3].

Theorem 1.4 Let $I \subseteq \mathbb{R}$ be an interval, and let $f: I \rightarrow \mathbb{R}$ be a convex function. Let $\Phi:[a, b] \rightarrow I$ be such that $f \circ \Phi$ is also convex, where $a<b$. Then for $n \in \mathbb{N}, \lambda_{0}=0, \lambda_{n+1}=1$, and arbitrary $0 \leq \lambda_{1} \leq \cdots \leq \lambda_{n} \leq 1$, we have

$$
\begin{aligned}
f\left(\frac{1}{b-a} \int_{a}^{b} \Phi(x) d x\right) & \leq l\left(\lambda_{1}, \ldots, \lambda_{n}\right) \leq \frac{1}{b-a} \int_{a}^{b} f \circ \Phi(x) d x \\
& \leq L\left(\lambda_{1}, \ldots, \lambda_{n}\right) \leq \frac{f \circ \Phi(a)+f \circ \Phi(b)}{2},
\end{aligned}
$$

where

$$
l\left(\lambda_{1}, \ldots, \lambda_{n}\right)=\sum_{k=0}^{n}\left(\lambda_{k+1}-\lambda_{k}\right) f\left(\frac{1}{\left(\lambda_{k+1}-\lambda_{k}\right)(b-a)} \int_{\left(1-\lambda_{k}\right) a+\lambda_{k} b}^{\left(1-\lambda_{k+1}\right) a+\lambda_{k+1} b} \Phi(x) d x\right)
$$

and

$$
L\left(\lambda_{1}, \ldots, \lambda_{n}\right)=\sum_{k=0}^{n}\left(\lambda_{k+1}-\lambda_{k}\right) \frac{f \circ \Phi\left(\left(1-\lambda_{k}\right) a+\lambda_{k} b\right)+f \circ \Phi\left(\left(1-\lambda_{k+1}\right) a+\lambda_{k+1} b\right)}{2} .
$$

Applying the previous theorem to $\Phi(x)=x$ and $n=1$, we get inequality (1.6).

We also give a refinement of the Hermite-Hadamard inequality. In the last section, we give some interesting estimates for the integral Csiszár divergence and for its important particular cases.

\section{New refinements}

Our first result is a refinement of the integral form of the Jensen inequality (1.2).

Theorem 2.1 Let $g$ be an integrable function defined on an interval $[a, b]$, and let $a_{0}, a_{1}, \ldots, a_{n-1}, a_{n}$ be such that $a=a_{0}<a_{1}<\cdots<a_{n-1}<a_{n}=b$. If $f$ is a convex function given on an interval $I$ that includes the image of $g$, then

$$
\begin{aligned}
f\left(\frac{1}{P(b)} \int_{a}^{b} p(t) g(t) d t\right) & \leq \frac{1}{P(b)} \sum_{i=1}^{n}\left(\int_{a_{i-1}}^{a_{i}} p(t) d t\right) f\left(\frac{\int_{a_{i-1}}^{a_{i}} p(t) g(t) d t}{\int_{a_{i-1}}^{a_{i}} p(t) d t}\right) \\
& \leq \frac{1}{P(b)} \int_{a}^{b} p(t) f(g(t)) d t
\end{aligned}
$$


where $p:[a, b] \rightarrow \mathbb{R}$ is a nonnegative function, and

$$
P(t)=\int_{a}^{t} p(x) d x
$$

Proof Let $a_{0}, a_{1}, \ldots, a_{n-1}, a_{n}$ be such that $a=a_{0}<a_{1}<\cdots<a_{n-1}<a_{n}=b$. Then we have (using Jensen's inequality)

$$
\begin{aligned}
f\left(\frac{1}{P(b)} \int_{a}^{b} p(t) g(t) d t\right) & =f\left(\frac{1}{P(b)} \sum_{i=1}^{n} \int_{a_{i-1}}^{a_{i}} p(t) g(t) d t\right) \\
& =f\left(\frac{1}{P(b)} \sum_{i=1}^{n}\left(\int_{a_{i-1}}^{a_{i}} p(t) d t\right) \frac{\int_{a_{i-1}}^{a_{i}} p(t) g(t) d t}{\int_{a_{i-1}}^{a_{i}} p(t) d t}\right) \\
& \leq \frac{1}{P(b)} \sum_{i=1}^{n}\left(\int_{a_{i-1}}^{a_{i}} p(t) d t\right) f\left(\frac{\int_{a_{i-1}}^{a_{i}} p(t) g(t) d t}{\int_{a_{i-1}}^{a_{i}} p(t) d t}\right),
\end{aligned}
$$

which is the left-hand side of (2.1).

Now we will use inequality (1.2) on each subinterval $\left[a_{i-1}, a_{i}\right]$ :

$$
\begin{aligned}
& \frac{1}{P(b)} \sum_{i=1}^{n}\left(\int_{a_{i-1}}^{a_{i}} p(t) d t\right) f\left(\frac{1}{\int_{a_{i-1}}^{a_{i}} p(t) d t} \int_{a_{i-1}}^{a_{i}} p(t) g(t) d t\right) \\
& \quad \leq \frac{1}{P(b)} \sum_{i=1}^{n}\left(\int_{a_{i-1}}^{a_{i}} p(t) d t\right) \frac{1}{\int_{a_{i-1}}^{a_{i}} p(t) d t} \int_{a_{i-1}}^{a_{i}} p(t) f(g(t) d t,
\end{aligned}
$$

which is the right-hand side of (2.1).

The next result is a refinement of the integral form of the Lah-Ribarič inequality (1.3). We need the following lemma.

Lemma 2.2 If $f$ is a convex function on an interval $I$, then for $a, b, u, c, d \in I$ such that $a \leq b \leq u \leq c \leq d, b<c$, we have

$$
\frac{c-u}{c-b} f(b)+\frac{u-b}{c-b} f(c) \leq \frac{d-u}{d-a} f(a)+\frac{u-a}{d-a} f(d) .
$$

Proof We can write

$$
\begin{aligned}
& b=\frac{d-b}{d-a} a+\frac{b-a}{d-a} d, \\
& c=\frac{d-c}{d-a} a+\frac{c-a}{d-a} d,
\end{aligned}
$$

and since $f$ is convex,

$$
\begin{aligned}
f(b) & \leq \frac{d-b}{d-a} f(a)+\frac{b-a}{d-a} f(d), \\
f(c) & \leq \frac{d-c}{d-a} f(a)+\frac{c-a}{d-a} f(d) .
\end{aligned}
$$


Now we have

$$
\begin{aligned}
& \frac{c-u}{c-b} f(b)+\frac{u-b}{c-b} f(c) \\
& \quad \leq \frac{c-u}{c-b}\left[\frac{d-b}{d-a} f(a)+\frac{b-a}{d-a} f(d)\right]+\frac{u-b}{c-b}\left[\frac{d-c}{d-a} f(a)+\frac{c-a}{d-a} f(d)\right] \\
& \quad=\frac{d-u}{d-a} f(a)+\frac{u-a}{d-a} f(d) .
\end{aligned}
$$

Theorem 2.3 Let $g$ be an integrable function defined on an interval $[a, b]$, and let $a_{0}, a_{1}, \ldots, a_{n-1}, a_{n}$ be such that $a=a_{0}<a_{1}<\cdots<a_{n-1}<a_{n}=b$ and $m_{i} \leq g(t) \leq M_{i}$ for $t \in\left[a_{i-1}, a_{i}\right], m_{i}<M_{i}, i=1, \ldots, n, m=\min _{i \in\{1, \ldots, n\}} m_{i}$, and $M=\max _{i \in\{1, \ldots, n\}} M_{i}$. Iff is a convex function given on an interval I that includes the image of $g$, then

$$
\begin{aligned}
& \frac{1}{P(b)} \int_{a}^{b} p(t) f(g(t)) d t \\
& \quad \leq \frac{1}{P(b)} \sum_{i=1}^{n} p_{i}\left[\frac{M_{i}-\bar{g}_{i}}{M_{i}-m_{i}} f\left(m_{i}\right)+\frac{\bar{g}_{i}-m_{i}}{M_{i}-m_{i}} f\left(M_{i}\right)\right] \\
& \quad \leq \frac{M-\bar{g}}{M-m} f(m)+\frac{\bar{g}-m}{M-m} f(M),
\end{aligned}
$$

where $p:[a, b] \rightarrow \mathbb{R}$ is nonnegative function,

$$
P(t)=\int_{a}^{t} p(x) d x
$$

and $\bar{g}, \bar{g}_{i}, p_{i}$ are defined as

$$
\bar{g}=\frac{\int_{a}^{b} p(t) g(t) d t}{P(b)}, \quad \bar{g}_{i}=\frac{\int_{a_{i-1}}^{a_{i}} p(t) g(t) d t}{\int_{a_{i-1}}^{a_{i}} p(t) d t}, \quad p_{i}=\int_{a_{i-1}}^{a_{i}} p(t) d t
$$

Proof We will use (1.3) on each subinterval $\left[a_{i-1}, a_{i}\right]$ :

$$
\begin{aligned}
& \frac{1}{P(b)} \int_{a}^{b} p(t) f(g(t)) d t \\
& \quad=\frac{1}{P(b)} \sum_{i=1}^{n} \int_{a_{i-1}}^{a_{i}} p(t) f(g(t)) d t \\
& \quad \leq \frac{1}{P(b)} \sum_{i=1}^{n}\left(\int_{a_{i-1}}^{a_{i}} p(t) d t\right)\left[\frac{M_{i}-\frac{\int_{a_{i-1}}^{a_{i}} p(t) g(t) d t}{\int_{a_{i-1}}^{a_{i}} p(t) d t}}{M_{i}-m_{i}} f\left(m_{i}\right)+\frac{\frac{\int_{a_{i-1}}^{a_{i}} p(t) g(t) d t}{\int_{a_{i-1}}^{a_{i}} p(t) d t}-m_{i}}{M_{i}-m_{i}} f\left(M_{i}\right)\right],
\end{aligned}
$$

which is the left-hand side of inequality (2.2).

Using $m \leq m_{i} \leq \bar{g}_{i} \leq M_{i} \leq M, m<M, m_{i}<M_{i}$, and Lemma 2.2, we get

$$
\begin{aligned}
& \frac{1}{P(b)} \sum_{i=1}^{n} p_{i}\left[\frac{M_{i}-\bar{g}_{i}}{M_{i}-m_{i}} f\left(m_{i}\right)+\frac{\bar{g}_{i}-m_{i}}{M_{i}-m_{i}} f\left(M_{i}\right)\right] \\
& \quad \leq \frac{1}{P(b)} \sum_{i=1}^{n}\left[\frac{p_{i} M-\int_{a_{i-1}}^{a_{i}} p(t) g(t) d t}{M-m} f(m)+\frac{\int_{a_{i-1}}^{a_{i}} p(t) g(t) d t-p_{i} m}{M-m} f(M)\right]
\end{aligned}
$$




$$
\begin{aligned}
= & \frac{1}{P(b)}\left[\frac{\sum_{i=1}^{n} p_{i} M-\sum_{i=1}^{n} \int_{a_{i-1}}^{a_{i}} p(t) g(t) d t}{M-m} f(m)\right. \\
& \left.+\frac{\sum_{i=1}^{n} \int_{a_{i-1}}^{a_{i}} p(t) g(t) d t-\sum_{i=1}^{n} p_{i} m}{M-m} f(M)\right] \\
= & \frac{M-\frac{\int_{a}^{b} p(t) g(t) d t}{P(b)}}{M-m} f(m)+\frac{\frac{\int_{a}^{b} p(t) g(t) d t}{P(b)}-m}{M-m} f(M),
\end{aligned}
$$

which is the right-hand side of (2.2).

Remark 2.4 If we set $p(t)=1$ in Theorem 2.1, then we get (1.7) in the form

$$
\begin{aligned}
f\left(\frac{1}{b-a} \int_{a}^{b} g(t) d t\right) & \leq \frac{1}{b-a} \sum_{i=1}^{n}\left(a_{i}-a_{i-1}\right) f\left(\frac{\int_{a_{i-1}}^{a_{i}} g(t) d t}{a_{i}-a_{i-1}}\right) \\
& \leq \frac{1}{b-a} \int_{a}^{b} f(g(t)) d t
\end{aligned}
$$

In particular, for $g(t)=t$, this gives

$$
f\left(\frac{a+b}{2}\right) \leq \frac{1}{b-a} \sum_{i=1}^{n}\left(a_{i}-a_{i-1}\right) f\left(\frac{a_{i-1}+a_{i}}{2}\right) \leq \frac{1}{b-a} \int_{a}^{b} f(t) d t
$$

which is a refinement of the left-hand side of (1.6).

Analogously, from Theorem 2.3 we have (for $p(t)=1$ )

$$
\begin{aligned}
& \frac{1}{b-a} \int_{a}^{b} f(g(t)) d t \\
& \quad \leq \frac{1}{b-a} \sum_{i=1}^{n}\left(a_{i}-a_{i-1}\right)\left[\frac{M_{i}-\frac{\int_{a_{i-1}}^{a_{i}} g(t) d t}{a_{i}-a_{i-1}}}{M_{i}-m_{i}} f\left(m_{i}\right)+\frac{\frac{\int_{a_{i-1}}^{a_{i}} g(t) d t}{a_{i}-a_{i-1}}-m_{i}}{M_{i}-m_{i}} f\left(M_{i}\right)\right] \\
& \quad \leq \frac{M-\frac{\int_{a}^{b} g(t) d t}{b-a}}{M-m} f(m)+\frac{\frac{\int_{a}^{b} g(t) d t}{b-a}-m}{M-m} f(M),
\end{aligned}
$$

and for $g(t)=t, m_{i}=a_{i-1}, M_{i}=a_{i}$, we get

$$
\begin{aligned}
& \frac{1}{b-a} \int_{a}^{b} f(t) d t \\
& \leq \frac{1}{b-a} \sum_{i=1}^{n}\left(a_{i}-a_{i-1}\right)\left[\frac{a_{i}-\frac{a_{i-1}+a_{i}}{2}}{a_{i}-a_{i-1}} f\left(a_{i-1}\right)+\frac{\frac{a_{i-1}+a_{i}}{2}-a_{i-1}}{a_{i}-a_{i-1}} f\left(a_{i}\right)\right] \\
& \quad=\frac{1}{b-a} \sum_{i=1}^{n}\left(a_{i}-a_{i-1}\right) \frac{f\left(a_{i-1}\right)+f\left(a_{i}\right)}{2} \\
& \quad \leq \frac{f(a)+f(b)}{2},
\end{aligned}
$$

which is a refinement of the right-hand side of (1.6).

Using our main result, we give a refinement of the Hölder inequality (more about the Hölder inequality see [16]). 
Corollary 2.5 Let $p, q \in \mathbb{R}$ be such that $\frac{1}{p}+\frac{1}{q}=1$. Let $w, g_{1}$, and $g_{2}$ be nonnegative functions defined on $[a, b]$ such that $w g_{1}^{p}, w g_{2}^{q}, w g_{1} g_{2} \in L^{1}([a, b])$.

(i) If $p>1$, then

$$
\begin{aligned}
& \int_{a}^{b} w(t) g_{1}(t) g_{2}(t) d t \\
& \leq\left(\int_{a}^{b} w(t) g_{2}^{q}(t) d t\right)^{\frac{1}{q}} \\
& \quad \times\left(\sum_{i=1}^{n}\left(\int_{a_{i-1}}^{a_{i}} w(t) g_{2}^{q}(t) d t\right)^{1-p}\left(\int_{a_{i-1}}^{a_{i}} w(t) g_{1}(t) g_{2}(t) d t\right)^{p}\right)^{\frac{1}{p}} \\
& \leq\left(\int_{a}^{b} w(t) g_{1}^{p}(t) d t\right)^{\frac{1}{p}}\left(\int w(t) g_{2}^{q}(t) d t\right)^{\frac{1}{q}} .
\end{aligned}
$$

(ii) If $p<1, p \neq 0$, then

$$
\begin{aligned}
& \left(\int_{a}^{b} w(t) g_{1}^{p}(t) d t\right)^{\frac{1}{p}}\left(\int w(t) g_{2}^{q}(t) d t\right)^{\frac{1}{q}} \\
& \quad \leq \sum_{i=1}^{n}\left(\int_{a_{i-1}}^{a_{i}} w(t) g_{1}^{p}(t) d t\right)^{\frac{1}{p}}\left(\int_{a_{i-1}}^{a_{i}} w(t) g_{2}^{q}(t) d t\right)^{\frac{1}{q}} \\
& \quad \leq \int_{a}^{b} w(t) g_{1}(t) g_{2}(t) d t .
\end{aligned}
$$

Proof For the case $p>1$, we use Theorem 2.1 with $p(t)=w(t) g_{2}^{q}(t), g(t)=g_{1}(t) g_{2}^{-\frac{q}{p}}$, and the function $f(x)=x^{p}$, which is convex for $x>0, p>1$. From (2.1) we get

$$
\begin{aligned}
& \left(\frac{1}{\int_{a}^{b} w(t) g_{2}^{q}(t) d t} \int_{a}^{b} w(t) g_{2}^{q}(t) g_{1}(t) g_{2}^{-\frac{q}{p}} d t\right)^{p} \\
& \quad \leq \frac{1}{\int_{a}^{b} w(t) g_{2}^{q}(t) d t} \sum_{i=1}^{n}\left(\int_{a_{i-1}}^{a_{i}} w(t) g_{2}^{q}(t) d t\right)\left(\frac{\int_{a_{i-1}}^{a_{i}} w(t) g_{2}^{q}(t) g_{1}(t) g_{2}^{-\frac{q}{p}}(t) d t}{\int_{a_{i-1}}^{a_{i}} w(t) g_{2}^{q}(t) d t}\right)^{p} \\
& \quad \leq \frac{1}{\int_{a}^{b} w(t) g_{2}^{q}(t) d t} \int_{a}^{b} w(t) g_{2}^{q}(t)\left(g_{1}(t) g_{2}^{-\frac{q}{p}}(t)\right)^{p} d t .
\end{aligned}
$$

Using $q-\frac{q}{p}=1$, multiplying by $\int_{a}^{b} w(t) g_{2}^{q}(t) d t$, and taking the power $\frac{1}{p}$, we have

$$
\begin{aligned}
& \left(\int_{a}^{b} w(t) g_{2}^{q}(t) d t\right)^{\frac{1}{p}-1}\left(\int_{a}^{b} w(t) g_{1}(t) g_{2}(t) d t\right) \\
& \quad \leq\left(\sum_{i=1}^{n}\left(\int_{a_{i-1}}^{a_{i}} w(t) g_{2}^{q}(t) d t\right)^{1-p}\left(\int_{a_{i-1}}^{a_{i}} w(t) g_{1}(t) g_{2}(t) d t\right)^{p}\right)^{\frac{1}{p}} \\
& \quad \leq\left(\int_{a}^{b} w(t) g_{1}^{p}(t) d t\right)^{\frac{1}{p}} .
\end{aligned}
$$


Now multiplying by $\left(\int_{a}^{b} w(t) g_{2}^{q}(t) d t\right)^{\frac{1}{q}}$, we get

$$
\begin{aligned}
& \int_{a}^{b} w(t) g_{1}(t) g_{2}(t) d t \\
& \quad \leq\left(\int_{a}^{b} w(t) g_{2}^{q}(t) d t\right)^{\frac{1}{q}}\left(\sum_{i=1}^{n}\left(\int_{a_{i-1}}^{a_{i}} w(t) g_{2}^{q}(t) d t\right)^{1-p}\left(\int_{a_{i-1}}^{a_{i}} w(t) g_{1}(t) g_{2}(t) d t\right)^{p}\right)^{\frac{1}{p}} \\
& \quad \leq\left(\int_{a}^{b} w(t) g_{2}^{q}(t) d t\right)^{\frac{1}{q}}\left(\int_{a}^{b} w(t) g_{1}^{p}(t) d t\right)^{\frac{1}{p}} .
\end{aligned}
$$

For $0<p<1$, we use Theorem 2.1 with $p(t)=w(t) g_{2}^{q}(t), g(t)=g_{1}^{p}(t) g_{2}^{-q}(t)$, and the function $f(x)=x^{\frac{1}{p}}$, which is convex for $x>0,0<p<1$. From (2.1) we get

$$
\begin{aligned}
& \left(\frac{1}{\int_{a}^{b} w(t) g_{2}^{q}(t) d t} \int_{a}^{b} w(t) g_{2}^{q}(t) g_{1}^{p}(t) g_{2}^{-q} d t\right)^{\frac{1}{p}} \\
& \quad \leq \frac{1}{\int_{a}^{b} w(t) g_{2}^{q}(t) d t} \sum_{i=1}^{n}\left(\int_{a_{i-1}}^{a_{i}} w(t) g_{2}^{q}(t) d t\right)\left(\frac{\int_{a_{i-1}}^{a_{i}} w(t) g_{2}^{q}(t) g_{1}^{p}(t) g_{2}^{-q} d t}{\int_{a_{i-1}}^{a_{i}} w(t) g_{2}^{q}(t) d t}\right)^{\frac{1}{p}} \\
& \quad \leq \frac{1}{\int_{a}^{b} w(t) g_{2}^{q}(t) d t} \int_{a}^{b} w(t) g_{2}^{q}(t)\left(g_{1}^{p}(t) g_{2}^{-q}\right)^{\frac{1}{p}} d t .
\end{aligned}
$$

Now using $q-\frac{q}{p}=1$ and multiplying by $\int_{a}^{b} w(t) g_{2}^{q}(t) d t$, we have

$$
\begin{aligned}
& \left(\int_{a}^{b} w(t) g_{1}^{p}(t) d t\right)^{\frac{1}{p}}\left(\int w(t) g_{2}^{q}(t) d t\right)^{\frac{1}{q}} \\
& \quad \leq \sum_{i=1}^{n}\left(\int_{a_{i-1}}^{a_{i}} w(t) g_{2}^{q}(t) d t\right)^{\frac{1}{q}}\left(\int_{a_{i-1}}^{a_{i}} w(t) g_{1}^{p}(t) d t\right)^{\frac{1}{p}} \\
& \quad \leq \int_{a}^{b} w(t) g_{1}(t) g_{2}(t) d t .
\end{aligned}
$$

If $p<0$, then $0<q<1$, and we have the same result by symmetry.

Let $p$ and $g$ be positive integrable functions defined on $[a, b]$. Then the integral power means of order $r \in \mathbb{R}$ are defined as follows:

$$
M_{r}(g ; p ; a, b)= \begin{cases}\left(\frac{1}{\int_{a}^{b} p(x) d x} \int_{a}^{b} p(x) g^{r}(x) d x\right)^{\frac{1}{r}}, & r \neq 0, \\ \exp \left(\frac{\int_{a}^{b} p(x) \log g(x) d x}{\int_{a}^{b} p(x) d x}\right), & r=0 .\end{cases}
$$

Let $\mathbf{x}=\left(x_{1}, \ldots, x_{n}\right)$ and $\mathbf{w}=\left(w_{1}, \ldots, w_{n}\right)$ be positive $n$-tuples. The weighted power mean (of the $n$-tuple $\mathbf{x}$ with weight $\mathbf{w}$ ) of order $r \in \mathbb{R}$ is defined as

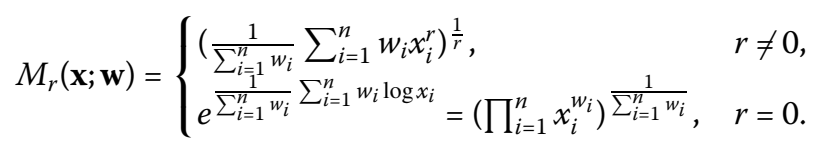

In this paper, it is more suitable to use the notation $M_{r}\left(x_{i} ; w_{i} ; \overline{1, n}\right)$. 
Using our main result, we obtain following inequalities for integral power means.

Corollary 2.6 Let $p$ and $g$ be positive integrable functions defined on $[a, b]$, and let $a_{0}, a_{1}, \ldots, a_{n-1}, a_{n}$ be such that $a=a_{0}<a_{1}<\cdots<a_{n-1}<a_{n}=b$. Let $s, t \in \mathbb{R}$ be such that $s \leq t$. Then

$$
\begin{aligned}
M_{s}(g ; p ; a, b) & \leq M_{t}\left(M_{s}\left(g ; p ; a_{i-1}, a_{i}\right) ; \int_{a_{i-1}}^{a_{i}} p(x) d x ; \overline{1, n}\right) \\
& \leq M_{t}(g ; p ; a . b), \\
M_{t}(g ; p ; a, b) & \geq M_{s}\left(M_{t}\left(g ; p ; a_{i-1}, a_{i}\right) ; \int_{a_{i-1}}^{a_{i}} p(x) d x ; \overline{1, n}\right) \\
& \geq M_{s}(g ; p ; a . b) .
\end{aligned}
$$

Proof We use Theorem 2.1 with $f(x)=x^{\frac{t}{s}}$ for $x>0, s, t \in \mathbb{R}, s, t \neq 0, s \leq t$ (convex on $\langle 0,+\infty\rangle)$. From (2.1) we get

$$
\begin{aligned}
\left(\frac{1}{P(b)} \int_{a}^{b} p(x) g(x) d x\right)^{\frac{t}{s}} & \leq \frac{1}{P(b)} \sum_{i=1}^{n}\left(\int_{a_{i-1}}^{a_{i}} p(x) d x\right)\left(\frac{1}{\int_{a_{i-1}}^{a_{i}} p(x) d x} \int_{a_{i-1}}^{a_{i}} p(x) g(x) d x\right)^{\frac{t}{s}} \\
& \leq \frac{1}{P(b)} \int_{a}^{b} p(x) g^{\frac{t}{s}}(x) d x
\end{aligned}
$$

Substituting $g$ with $g^{s}$ and taking the power $\frac{1}{t}$, we get the result.

Similarly, we use Theorem 2.1 with $f(x)=x^{\frac{s}{t}}$ for $x>0, s, t \in \mathbb{R}, s, t \neq 0, s \leq t$ (concave on $\langle 0,+\infty\rangle)$. From (2.1) we get

$$
\begin{aligned}
\left(\frac{1}{P(b)} \int_{a}^{b} p(x) g(x) d x\right)^{\frac{s}{t}} & \geq \frac{1}{P(b)} \sum_{i=1}^{n}\left(\int_{a_{i-1}}^{a_{i}} p(x) d x\right)\left(\frac{1}{\int_{a_{i-1}}^{a_{i}} p(x) d x} \int_{a_{i-1}}^{a_{i}} p(x) g(x) d x\right)^{\frac{s}{t}} \\
& \geq \frac{1}{P(b)} \int_{a}^{b} p(x) g^{\frac{s}{t}}(x) d x
\end{aligned}
$$

Substituting $g$ with $g^{t}$ and takingthe power $\frac{1}{s}$, we get the result.

The cases $t=0$ and $s=0$ follow from inequalities (2.3) and (2.4) by simple limiting process.

Means of the type

$$
M_{t}\left(M_{s}\left(g ; p ; a_{i-1}, a_{i}\right) ; \int_{a_{i-1}}^{a_{i}} p(x) d x ; \overline{1, n}\right)
$$

can be regarded as mixed means.

Let $p$ be positive integrable function defined on $[a, b]$, and let $g$ be any integrable function defined on $[a, b]$. Then for a strictly monotone continuous function $h$ with domain belonging to the image of $g$, the quasiarithmetic mean is defined as follows:

$$
M_{h}(g ; p ; a, b)=h^{-1}\left(\frac{1}{\int_{a}^{b} p(x) d x} \int_{a}^{b} p(x) h(g(x)) d x\right) .
$$


Using our main result, we obtain following inequalities for quasiarithmetic means.

Corollary 2.7 Let $p$ be positive integrable function defined on $[a, b]$, let $g$ be an integrable function defined on $[a, b]$, and let $a_{0}, a_{1}, \ldots, a_{n-1}, a_{n}$ be such that $a=a_{0}<a_{1}<\cdots<a_{n-1}<$ $a_{n}=b$. Also, assume that $h$ is a strictly monotone continuous function with domain belonging to the image of $g$. If $\circ \circ h^{-1}$ is a convex function, then

$$
\begin{aligned}
f\left(M_{h}(g ; p ; a, b)\right) & \leq \frac{1}{P(b)} \sum_{i=1}^{n}\left(\int_{a_{i-1}}^{a_{i}} p(x) d x\right) f\left(M_{h}\left(g ; p ; a_{i-1}, a_{i}\right)\right. \\
& \leq \frac{1}{P(b)} \int_{a}^{b} p(x) f(g(x)) d x .
\end{aligned}
$$

Proof We use Theorem 2.1 with $f \rightarrow f \circ h^{-1}$ and $g \rightarrow h \circ g$.

\section{Applications in information theory}

In this section, we give some interesting estimates for the integral Csiszár divergence and for its important particular cases (see, e.g., $[4,5,9,10,12,15]$ ).

Definition 3.1 (Csiszár divergence) Let $f: I \rightarrow \mathbb{R}$ be a function defined on some positive interval $I$, and let $p, q:[a, b] \rightarrow \mathbb{R}^{+}$be two probability density functions such that $\frac{p(t)}{q(t)} \in I$ for $t \in[a, b]$. The Csiszár divergence is defined as

$$
C_{d}(p, q)=\int_{a}^{b} q(t) f\left(\frac{p(t)}{q(t)}\right) d t
$$

Theorem 3.2 Let $f: I \rightarrow \mathbb{R}$ be a convex function defined on a positive interval I, let $p, q:[a, b] \rightarrow \mathbb{R}^{+}$be probability density functions such that $\frac{p(t)}{q(t)} \in I$ for $t \in[a, b]$, and let $a_{0}, a_{1}, \ldots, a_{n-1}, a_{n}$ be such that $a=a_{0}<a_{1}<\cdots<a_{n-1}<a_{n}=b$. Then

$$
f(1) \leq \sum_{i=1}^{n}\left(\int_{a_{i-1}}^{a_{i}} q(t) d t\right) f\left(\frac{\int_{a_{i-1}}^{a_{i}} p(t) d t}{\int_{a_{i-1}}^{a_{i}} q(t) d t}\right) \leq C_{d}(p, q) .
$$

Proof Using Theorem 2.1 with $p \rightarrow q$ and $g \rightarrow \frac{p}{q}$, we obtain the result.

The condition $\frac{p(t)}{q(t)} \in I$ for $t \in[a, b]$ obviously implies that $1 \in I$ and $\frac{\int_{a_{i-1}}^{a_{i}} p(t) d t}{\iint_{i-1}^{a_{i}} q(t) d t} \in I$ for $i=1, \ldots, n$.

Theorem 3.3 Let $f: I \rightarrow \mathbb{R}$ be a convex function defined on a positive interval I, let $p, q:[a, b] \rightarrow \mathbb{R}^{+}$be probability density functions such that $\frac{p(t)}{q(t)} \in I$ for $t \in[a, b]$, and let $a_{0}, a_{1}, \ldots, a_{n-1}, a_{n}$ be such that $a=a_{0}<a_{1}<\cdots<a_{n-1}<a_{n}=b$. Let $m_{i} \leq \frac{p(t)}{q(t)} \leq M_{i}$ for $t \in\left[a_{i-1}, a_{i}\right], m_{i}<M_{i}, i=1, \ldots, n, m=\min _{i=1, \ldots, n} m_{i}$, and $M=\max _{i=1, \ldots, n} M_{i}$. Then

$$
\begin{aligned}
& C_{d}(p, q) \\
& \quad \leq \sum_{i=1}^{n}\left(\int_{a_{i-1}}^{a_{i}} q(t) d t\right)\left[\frac{M_{i}-\frac{\int_{a_{i-1}}^{a_{i}} p(t) d t}{\int_{a_{i-1}}^{a_{i}} q(t) d t}}{M_{i}-m_{i}} f\left(m_{i}\right)+\frac{\frac{\int_{a_{i-1}}^{a_{i}} p(t) d t}{\int_{a_{i-1}}^{a_{i}} q(t) d t}-m_{i}}{M_{i}-m_{i}} f\left(M_{i}\right)\right] \\
& \quad \leq \frac{M-1}{M-m} f(m)+\frac{1-m}{M-m} f(M) .
\end{aligned}
$$


Proof Using Theorem 2.3 with $p \rightarrow q$ and $g \rightarrow \frac{p}{q}$, we obtain the result.

Definition 3.4 (Shannon entropy) Let $p:[a, b] \rightarrow \mathbb{R}^{+}$be a probability density function. The Shannon entropy is defined as

$$
\mathrm{SE}(p)=-\int_{a}^{b} p(t) \log p(t) d t
$$

Corollary 3.5 Let $q:[a, b] \rightarrow \mathbb{R}^{+}$be a probability density function, and let $a_{0}, a_{1}, \ldots$, $a_{n-1}, a_{n}$ be such that $a=a_{0}<a_{1}<\cdots<a_{n-1}<a_{n}=b$. Then

$$
-\log (b-a) \leq \sum_{i=1}^{n}\left(\int_{a_{i-1}}^{a_{i}} q(t) d t\right) \log \left(\frac{\int_{a_{i-1}}^{a_{i}} q(t) d t}{a_{i}-a_{i-1}}\right) \leq-\operatorname{SE}(q)
$$

Proof Using Theorem 3.2 with $f(t)=-\log t, t \in \mathbb{R}^{+}$, and $p(t)=\frac{1}{b-a}, t \in[a, b]$, we obtain the result.

Corollary 3.6 Let $q:[a, b] \rightarrow \mathbb{R}^{+}$be a probability density function, let $a_{0}, a_{1}, \ldots, a_{n-1}, a_{n}$ be such that $a=a_{0}<a_{1}<\cdots<a_{n-1}<a_{n}=b$, and let $m_{i} \leq \frac{1}{q(t)} \leq M_{i}$ for $t \in\left[a_{i-1}, a_{i}\right], m_{i}<$ $M_{i}, i=1, \ldots, n, m=\min _{i=1, \ldots, n} m_{i}$ and $M=\max _{i=1, \ldots, n} M_{i}$. Then

$$
\begin{aligned}
& -\mathrm{SE}(q)+\log (b-a) \\
& \quad \leq \sum_{i=1}^{n}\left(\int_{a_{i-1}}^{a_{i}} q(t) d t\right)\left[\frac{\frac{a_{i}-a_{i-1}}{(b-a) \int_{a_{i-1}}^{a_{i}} q(t) d t}-M_{i}}{M_{i}-m_{i}} \log m_{i}+\frac{m_{i}-\frac{a_{i}-a_{i-1}}{(b-a) \int_{a_{i-1}}^{a_{i}} q(t) d t}}{M_{i}-m_{i}} \log M_{i}\right] \\
& \quad \leq \frac{1-M}{M-m} \log m+\frac{m-1}{M-m} \log M .
\end{aligned}
$$

Proof Using Theorem 3.3 with $f(t)=-\log t, t \in \mathbb{R}^{+}$, and $p(t)=\frac{1}{b-a}, t \in[a, b]$, we obtain the result.

Definition 3.7 (Kullback-Leibler divergence) Let $p, q:[a, b] \rightarrow \mathbb{R}^{+}$be two probability density functions. The Kullback-Leibler divergence is defined as

$$
\mathrm{KL}_{d}(p, q)=\int_{a}^{b} p(t) \log \left(\frac{p(t)}{q(t)}\right) d t
$$

Corollary 3.8 Let $p, q:[a, b] \rightarrow \mathbb{R}^{+}$be probability density functions, and let $a_{0}, a_{1}, \ldots$, $a_{n-1}, a_{n}$ be such that $a=a_{0}<a_{1}<\cdots<a_{n-1}<a_{n}=b$. Then

$$
0 \leq \sum_{i=1}^{n}\left(\int_{a_{i-1}}^{a_{i}} p(t) d t\right) \log \left(\frac{\int_{a_{i-1}}^{a_{i}} p(t) d t}{\int_{a_{i-1}}^{a_{i}} q(t) d t}\right) \leq \mathrm{KL}_{d}(p, q)
$$

Proof Using Theorem 3.2 with $f(t)=t \log t, t \in \mathbb{R}^{+}$, we obtain the result.

Corollary 3.9 Let $p, q:[a, b] \rightarrow \mathbb{R}^{+}$be probability density functions, let $a_{0}, a_{1}, \ldots, a_{n-1}, a_{n}$ be such that $a=a_{0}<a_{1}<\cdots<a_{n-1}<a_{n}=b$, and let $m_{i} \leq \frac{1}{q(t)} \leq M_{i}$ for $t \in\left[a_{i-1}, a_{i}\right], m_{i}<$ 
$M_{i}, i=1, \ldots, n, m=\min _{i=1, \ldots, n} m_{i}$ and $M=\max _{i=1, \ldots, n} M_{i}$. Then

$$
\begin{aligned}
& \mathrm{KL}_{d}(p, q) \\
& \quad \leq \sum_{i=1}^{n}\left(\int_{a_{i-1}}^{a_{i}} q(t) d t\right)\left[\frac{M_{i}-\frac{\int_{a_{i-1}}^{a_{i}} p(t) d t}{\int_{a_{i-1}}^{a_{i}} q(t) d t}}{M_{i}-m_{i}} m_{i} \log m_{i}+\frac{\frac{\int_{a_{i-1}}^{a_{i}} p(t) d t}{\int_{a_{i-1}}^{a_{i-1}} q(t) d t}-m_{i}}{M_{i}-m_{i}} M_{i} \log M_{i}\right] \\
& \quad \leq \frac{M-1}{M-m} m \log m+\frac{1-m}{M-m} M \log M .
\end{aligned}
$$

Proof Using Theorem 3.3 with $f(t)=t \log t, t \in \mathbb{R}^{+}$, we obtain the result.

Definition 3.10 (Variational distance) Let $p, q:[a, b] \rightarrow \mathbb{R}^{+}$be two probability density functions. The variational distance is defined by

$$
V_{d}(p, q)=\int_{a}^{b}|p(t)-q(t)| d t
$$

The following corollary can be also proved elementarily using the triangle inequality for integrals.

Corollary 3.11 Let $p, q:[a, b] \rightarrow \mathbb{R}^{+}$be probability density functions, and let $a_{0}, a_{1}, \ldots$, $a_{n-1}, a_{n}$ be a such that $a=a_{0}<a_{1}<\cdots<a_{n-1}<a_{n}=b$. Then

$$
0 \leq \sum_{i=1}^{n}\left|\int_{a_{i-1}}^{a_{i}} p(t) d t-\int_{a_{i-1}}^{a_{i}} q(t) d t\right| \leq V_{d}(p, q)
$$

Proof Using Theorem 3.2 with $f(t)=|t-1|, t \in \mathbb{R}^{+}$, we obtain the result.

Corollary 3.12 Let $p, q:[a, b] \rightarrow \mathbb{R}^{+}$be probability density functions, let $a_{0}, a_{1}, \ldots, a_{n-1}, a_{n}$ be such that $a=a_{0}<a_{1}<\cdots<a_{n-1}<a_{n}=b$, and let $m_{i} \leq \frac{p(t)}{q(t)} \leq M_{i}$ for $t \in\left[a_{i-1}, a_{i}\right], m_{i}<$ $M_{i}, i=1, \ldots, n, m=\min _{i=1, \ldots, n} m_{i}$ and $M=\max _{i=1, \ldots, n} M_{i}$. Then

$$
\begin{aligned}
\int_{a}^{b} & |p(t)-q(t)| d t \\
\leq & \sum_{i=1}^{n}\left[\frac{M_{i} \int_{a_{i-1}}^{a_{i}} q(t) d t-\int_{a_{i-1}}^{a_{i}} p(t) d t}{M_{i}-m_{i}}\left|m_{i}-1\right|\right. \\
& \left.\quad+\frac{\int_{a_{i-1}}^{a_{i}} p(t) d t-m_{i} \int_{a_{i-1}}^{a_{i}} q(t) d t}{M_{i}-m_{i}}\left|M_{i}-1\right|\right] \\
& \leq \frac{2(M-1)(1-m)}{M-m} .
\end{aligned}
$$

Proof Using Theorem 3.3 with $f(t)=|t-1|, t \in \mathbb{R}^{+}$, and $m \leq 1 \leq M$, we obtain get the result.

Definition 3.13 (Jeffrey's distance) Let $p, q:[a, b] \rightarrow \mathbb{R}^{+}$be two probability density functions. The Jeffrey distance is defined as

$$
J_{d}(p, q)=\int_{a}^{b}(p(t)-q(t)) \log \left(\frac{p(t)}{q(t)}\right) d t .
$$


Corollary 3.14 Let $p, q:[a, b] \rightarrow \mathbb{R}^{+}$be probability density functions, and let $a_{0}, a_{1}, \ldots$, $a_{n-1}, a_{n}$ be such that $a=a_{0}<a_{1}<\cdots<a_{n-1}<a_{n}=b$. Then

$$
\begin{aligned}
0 & \leq \sum_{i=1}^{n}\left(\int_{a_{i-1}}^{a_{i}} p(t) d t-\int_{a_{i-1}}^{a_{i}} q(t) d t\right) \log \left(\frac{\int_{a_{i-1}}^{a_{i}} p(t) d t}{\int_{a_{i-1}}^{a_{i}} q(t) d t}\right) \\
& \leq J_{d}(p, q) .
\end{aligned}
$$

Proof Using Theorem 3.2 with $f(t)=(t-1) \log t, t \in \mathbb{R}^{+}$, we obtain the result.

Corollary 3.15 Let $p, q:[a, b] \rightarrow \mathbb{R}^{+}$be probability density functions, let $a_{0}, a_{1}, \ldots, a_{n-1}, a_{n}$ be such that $a=a_{0}<a_{1}<\cdots<a_{n-1}<a_{n}=b$, and let $m_{i} \leq \frac{p(t)}{q(t)} \leq M_{i}$ for $t \in\left[a_{i-1}, a_{i}\right], m_{i}<$ $M_{i}, i=1, \ldots, n, m=\min _{i=1, \ldots, n} m_{i}$, and $M=\max _{i=1, \ldots, n} M_{i}$. Then

$$
\begin{aligned}
J_{d}(p, q) & \\
\leq & \sum_{i=1}^{n}\left[\frac{M_{i} \int_{a_{i-1}}^{a_{i}} q(t) d t-\int_{a_{i-1}}^{a_{i}} p(t) d t}{M_{i}-m_{i}}\left(m_{i}-1\right) \log m_{i}\right. \\
& \left.+\frac{\int_{a_{i-1}}^{a_{i}} p(t) d t-m_{i} \int_{a_{i-1}}^{a_{i}} q(t) d t}{M_{i}-m_{i}}\left(M_{i}-1\right) \log M_{i}\right] \\
\leq & \frac{(M-1)(1-m)}{M-m} \log \frac{M}{m} .
\end{aligned}
$$

Proof Using Theorem 3.3 with $f(t)=(t-1) \log t, t \in \mathbb{R}^{+}$, we obtain the result.

Definition 3.16 (Bhattacharyya coefficient) Let $p, q:[a, b] \rightarrow \mathbb{R}^{+}$be two probability density functions. The Bhattacharyya distance is defined as

$$
B_{d}(p, q)=\int_{a}^{b} \sqrt{p(t) q(t)} d t .
$$

Corollary 3.17 Let $p, q:[a, b] \rightarrow \mathbb{R}^{+}$be probability density functions, and let $a_{0}, a_{1}, \ldots$, $a_{n-1}, a_{n}$ be such that $a=a_{0}<a_{1}<\cdots<a_{n-1}<a_{n}=b$. Then

$$
1 \geq \sum_{i=1}^{n} \sqrt{\int_{a_{i-1}}^{a_{i}} p(t) d t \int_{a_{i-1}}^{a_{i}} q(t) d t} \geq B_{d}(p, q) .
$$

Proof Using Theorem 3.2 with $f(t)=-\sqrt{t}, t \in \mathbb{R}^{+}$, we obtain the result.

Corollary 3.18 Let $p, q:[a, b] \rightarrow \mathbb{R}^{+}$be probability density functions, let $a_{0}, a_{1}, \ldots, a_{n-1}, a_{n}$ be such that $a=a_{0}<a_{1}<\cdots<a_{n-1}<a_{n}=b$, and let $m_{i} \leq \frac{p(t)}{q(t)} \leq M_{i}$ for $t \in\left[a_{i-1}, a_{i}\right], m_{i}<$ $M_{i}, i=1, \ldots, n, m=\min _{i=1, \ldots, n} m_{i}$, and $M=\max _{i=1, \ldots, n} M_{i}$. Then

$$
\begin{aligned}
& B_{d}(p, q) \\
& \quad \geq \sum_{i=1}^{n}\left[\frac{M_{i} \int_{a_{i-1}}^{a_{i}} q(t) d t-\int_{a_{i-1}}^{a_{i}} p(t) d t}{M_{i}-m_{i}} \sqrt{m_{i}}+\frac{\int_{a_{i-1}}^{a_{i}} p(t) d t-m_{i} \int_{a_{i-1}}^{a_{i}} q(t) d t}{M_{i}-m_{i}} \sqrt{M_{i}}\right] \\
& \quad \geq \frac{1+\sqrt{m M}}{\sqrt{m}+\sqrt{M}} .
\end{aligned}
$$


Proof Using Theorem 3.3 with $f(t)=-\sqrt{t}, t \in \mathbb{R}^{+}$, we obtain the result.

Definition 3.19 (Hellinger distance) Let $p, q:[a, b] \rightarrow \mathbb{R}^{+}$be two probability density functions. The Hellinger distance is defined as

$$
H_{d}(p, q)=\int_{a}^{b}(\sqrt{p(t)}-\sqrt{q(t)})^{2} d t
$$

Corollary 3.20 Let $p, q:[a, b] \rightarrow \mathbb{R}^{+}$be probability density functions, and let $a_{0}, a_{1}, \ldots$, $a_{n-1}, a_{n}$ be such that $a=a_{0}<a_{1}<\cdots<a_{n-1}<a_{n}=b$. Then

$$
0 \leq \sum_{i=1}^{n}\left(\sqrt{\int_{a_{i-1}}^{a_{i}} p(t) d t}-\sqrt{\int_{a_{i-1}}^{a_{i}} q(t) d t}\right)^{2} \leq H_{d}(p, q) .
$$

Proof Using Theorem 3.2 with $f(t)=(\sqrt{t}-1)^{2}, t \in \mathbb{R}^{+}$, we obtain the result.

Corollary 3.21 Let p, $q:[a, b] \rightarrow \mathbb{R}^{+}$be probability density functions, let $a_{0}, a_{1}, \ldots, a_{n-1}, a_{n}$ be such that $a=a_{0}<a_{1}<\cdots<a_{n-1}<a_{n}=b$, and let $m_{i} \leq \frac{p(t)}{q(t)} \leq M_{i}$ for $t \in\left[a_{i-1}, a_{i}\right], m_{i}<$ $M_{i}, i=1, \ldots, n, m=\min _{i=1, \ldots, n} m_{i}$, and $M=\max _{i=1, \ldots, n} M_{i}$. Then

$$
\begin{aligned}
& H_{d}(p, q) \\
& \quad \leq \sum_{i=1}^{n}\left[\frac{M_{i} \int_{a_{i-1}}^{a_{i}} q(t) d t-\int_{a_{i-1}}^{a_{i}} p(t) d t}{M_{i}-m_{i}}\left(\sqrt{m_{i}}-1\right)^{2}\right. \\
& \left.\quad+\frac{\int_{a_{i-1}}^{a_{i}} p(t) d t-m_{i} \int_{a_{i-1}}^{a_{i}} q(t) d t}{M_{i}-m_{i}}\left(\sqrt{M_{i}}-1\right)^{2}\right] \\
& \leq 2 \frac{(\sqrt{M}-1)(1-\sqrt{m})}{\sqrt{m}+\sqrt{M}} .
\end{aligned}
$$

Proof Using Theorem 3.3 with $f(t)=(\sqrt{t}-1)^{2}, t \in \mathbb{R}^{+}$, we obtain the result.

Definition 3.22 (Triangular discrimination) Let $p, q:[a, b] \rightarrow \mathbb{R}^{+}$be two probability density functions. The triangular discrimination between $p$ and $q$ is defined as

$$
T_{d}(p, q)=\int_{a}^{b} \frac{(p(t)-q(t))^{2}}{p(t)+q(t)} d t
$$

Corollary 3.23 Let p, $q:[a, b] \rightarrow \mathbb{R}^{+}$be probability density functions, and let $a_{0}, a_{1}, \ldots$, $a_{n-1}, a_{n}$ be such that $a=a_{0}<a_{1}<\cdots<a_{n-1}<a_{n}=b$. Then

$$
0 \leq \sum_{i=1}^{n} \frac{\left(\int_{a_{i-1}}^{a_{i}} p(t) d t-\int_{a_{i-1}}^{a_{i}} q(t) d t\right)^{2}}{\int_{a_{i-1}}^{a_{i}} p(t) d t+\int_{a_{i-1}}^{a_{i}} q(t) d t} \leq T_{d}(p, q) .
$$

Proof Using Theorem 3.2 with $f(t)=\frac{(t-1)^{2}}{t+1}, t \in \mathbb{R}^{+}$, we obtain the result.

Corollary 3.24 Let $p, q:[a, b] \rightarrow \mathbb{R}^{+}$be probability density functions, let $a_{0}, a_{1}, \ldots, a_{n-1}, a_{n}$ be such that $a=a_{0}<a_{1}<\cdots<a_{n-1}<a_{n}=b$, and let $m_{i} \leq \frac{p(t)}{q(t)} \leq M_{i}$ for $t \in\left[a_{i-1}, a_{i}\right], m_{i}<$ 
$M_{i}, i=1, \ldots, n, m=\min _{i=1, \ldots, n} m_{i}$, and $M=\max _{i=1, \ldots, n} M_{i}$. Then

$$
\begin{aligned}
& T_{d}(p, q) \\
& \leq \sum_{i=1}^{n}\left[\frac{M_{i} \int_{a_{i-1}}^{a_{i}} q(t) d t-\int_{a_{i-1}}^{a_{i}} p(t) d t}{M_{i}-m_{i}} \frac{\left(m_{i}-1\right)^{2}}{m_{i}+1}\right. \\
& \left.\quad+\frac{\int_{a_{i-1}}^{a_{i}} p(t) d t-m_{i} \int_{a_{i-1}}^{a_{i}} q(t) d t}{M_{i}-m_{i}} \frac{\left(M_{i}-1\right)^{2}}{M_{i}+1}\right] \\
& \leq \frac{2(M-1)(1-m)}{(M+1)(m+1)} .
\end{aligned}
$$

Proof Using Theorem 3.3 with $f(t)=\frac{(t-1)^{2}}{t+1}, t \in \mathbb{R}^{+}$, we obtain the result.

\section{Acknowledgements}

The research of the first author was supported by the Ministry of Education and Science of the Russian Federation (the Agreement number No. 02.a03.21.0008)

\section{Funding}

There is no funding for this work.

Availability of data and materials

Not applicable.

\section{Competing interests}

The authors declare that they have no competing interests.

\section{Authors' contributions}

Both authors jointly worked on the results, and they read and approved the final manuscript.

\section{Author details}

${ }^{1}$ RUDN University, Moscow, Russia. ${ }^{2}$ Department of Mathematics, Faculty of Science, University of Split, Split, Croatia.

\section{Publisher's Note}

Springer Nature remains neutral with regard to jurisdictional claims in published maps and institutional affiliations.

Received: 2 March 2020 Accepted: 3 April 2020 Published online: 21 April 2020

\section{References}

1. Dragomir, S.S., Khan, M.A., Abathun, A.: Refinement of Jensen's integral inequality. Open Math. 14, 221-228 (2016)

2. El Farissi, A.: Simple proof and refinement of Hermite-Hadamard inequality. J. Math. Inequal. 4(3), 365-369 (2010)

3. Gao, X:: A note on the Hermite-Hadamard inequality. J. Math. Inequal. 4(4), 587-591 (2010)

4. Ivelić Bradanović, S., Latif, N., Pečarić, Đ., Pečarić, J.: Sherman's and related inequalities with applications in information theory. J. Inequal. Appl. 2018, 98 (2018)

5. Jakšetić, J., Pečarić, Đ., Pečarić, J.: Hybrid Zipf-Mandelbrot law. J. Math. Inequal. 13, 275-286 (2019)

6. Jensen, J.L.W.V.: Om konvexe Funktioner og Uligheder mellem Middelvaerdier. Nyt Tidsskr. Math. 16B, 49-69 (1905) (German)

7. Khalid, S., On the refinements of the integral Jensen-Steffensen inequality. J. Inequal. Appl. 2013, 20 (2013) https://doi.org/10.1186/1029-242X-2013-20

8. Khan, M.A., Khan, J., Pečarić, J.: Generalization of Jensen's and Jensen-Steffensen's inequalities by generalized majorization theorem. J. Math. Inequal. 11(4), 1049-1074 (2017)

9. Khan, M.A., Pečarić, Đ., Pečarić, J.: Bounds for Csiszár divergence and hybrid Zipf-Mandelbrot entropy. Math. Methods Appl. Sci. 42(18), 7411-7424 (2019)

10. Khan, M.A., Pečarić, $Đ$., Pečarić, J.: New refinement of the Jensen inequality associated to certain functions with applications. J. Inequal. Appl. 2020, Article ID 76 (2020)

11. Lah, P., Ribarič, M.: Converse of Jensen's inequality for convex functions. Publ. Elektroteh. Fak. Univ. Beogr., Ser. Mat. Fiz. 412-460, 201-205 (1973)

12. Mikić, R., Pečarić, Đ., Pečarić, J.: Inequalities of the Jensen and Edmundson-Lah-Ribarič type for 3-convex functions with applications. J. Math. Inequal. 12, 677-692 (2018)

13. Mitrinović, D.S., Pečarić, J.E., Fink, A.M.: Classical and New Inequalities in Analysis. Mathematics and Its Applications (East European Series), vol. 61. Kluwer Academic, Dordrecht (1993). ISBN 0-7923-2064-6

14. Niculescu, C.P., Persson, L.-E.: Convex Functions and Their Applications. A Contemporary Approach. CMS Books in Mathematics/Ouvrages de Mathématiques de la SMC, vol. 23. Springer, New York (2006). ISBN 978-0387-24300-9 
15. Pečarić, Đ., Pečarić, J., Rodić, M.: On a Jensen-type inequality for generalized f-divergences and Zipf-Mandelbrot law. Math. Inequal. Appl. 22(4), 1463-1475 (2019)

16. Pečarić, J.E., Proschan, F., Tong, Y.L.: Convex Functions, Partial Orderings, and Statistical Applications. Mathematics in Science and Engineering, vol. 187. Academic Press, Boston (1992). ISBN 0-12-549250-2

Submit your manuscript to a SpringerOpen ${ }^{\circ}$ journal and benefit from:

- Convenient online submission

$\checkmark$ Rigorous peer review

- Open access: articles freely available online

- High visibility within the field

Retaining the copyright to your article

Submit your next manuscript at $\gg$ springeropen.com 